\title{
Dielectric Relaxation Losses in Lead Chloride and Lead Bromide: Localized Dipoles
}

\author{
W. E. VAN DEN BROM AND J. VOLGER \\ Solid State Department, Physical Laboratory, University of Utrecht, Sorbonnelaan 4, \\ Utrecht, The Netherlands
}

Received October 9, 1973

\begin{abstract}
A further analysis of previous reported measurements of dielectric relaxation losses in lead chloride and lead bromide crystals shows that the dipoles may occupy several energetically different positions, giving rise to localization of the dipoles and anomalous behaviour of the susceptibility. This energy difference is determined. Inspection of the crystal structure shows that the anion vacancy, being the mobile part of the dipole (which is an associate like $\left(\mathrm{Me}_{\mathbf{p b}} \cdot \mathrm{V}_{\mathbf{x}}\right)^{\mathrm{x}}$ or $\left(\mathrm{O}_{\mathbf{x}} \cdot \mathrm{V}_{\mathbf{x}}\right)^{\mathrm{x}}$ where $\mathrm{X}=\mathrm{Cl}, \mathrm{Br}$ ), may jump indeed between halide ion sites with different coordination number.

A comparison between the dipole concentration as calculated from the observed losses and the associate concentration as estimated from ionic conductivity experiments leads to the conclusion that local field corrections in the calculations are important.
\end{abstract}

\section{Introduction}

The properties of lead halides $\mathrm{PbCl}_{2}$ and $\mathrm{PbBr}_{2}$ and the effects related to the presence of defects in the crystals have been investigated extensively. In particular much work has been devoted to the optical properties like luminescence $(1,2)$, absorption (3) and photochemical decomposition (4), whereas electron spin resonance experiments $(1,5)$ gave insight into the structure of electronic centers and ionic conductivity studies into the mass transport $(6,7)$. All these experiments were performed on both pure and doped single crystals, grown in this laboratory from zone refined materials by the Bridgman technique (8).

The crystal structure of lead chloride (9) and lead bromide $(10)$ is orthorhombic with space group $D_{2 h}^{16}(P n m a)(1 I)$. The unit cell contains four formula units. The point group symmetry at a lead ion site is $C_{s}$. Every lead ion is surrounded by nine halide ions. Two types of halide ions can be discerned in the lattice: one type is surrounded by four and the other type by five lead ions (denoted by $X(1)$ and $X(2)$, respectively, with $\mathrm{X}=\mathrm{Cl}$ or $\mathrm{Br}$ ).

Copyright $\bigcirc 1974$ by Academic Press, Inc. All rights of reproduction in any form reserved.
Printed in Great Britain
The intrinsic point defects are of the Schottky type, i.e., cation and anion vacancies would be present in a stoichiometric amount, according to:

$$
\mathrm{PbX}_{2} \rightleftarrows \mathrm{V}_{\mathrm{Pb}}^{\prime \prime}+2 \mathrm{~V}_{\mathrm{X}}^{\prime}
$$

if thermal generation would be the only source. However, substitutional replacement of lead ions by aliovalent metal ions changes the situation according to the following electroneutrality conditions:

or

$$
\left[\mathrm{Me}_{\mathrm{Pb}}^{\prime}\right]+2\left[\mathrm{~V}_{\mathrm{Pb}}^{\prime \prime}\right]=\left[\mathrm{V}_{\mathrm{X}}^{\prime}\right]
$$

$$
\left[\mathrm{Me}_{\mathrm{Pb}}^{\cdot}\right]+\left[\mathrm{V}_{\mathrm{X}}^{\prime}\right]=2\left[\mathrm{~V}_{\mathrm{Pb}}^{\prime \prime}\right] \text {. }
$$

We use here the defect notation of Kröger (12) $\left(V_{A}\right.$ denotes a vacancy at the site of ion $A$, $\mathrm{Me}_{\mathrm{B}}$ a metal ion on the site of ion $\mathrm{B}$; square brackets denote concentrations and the superscripts ', ' and ${ }^{\times}$are effective negative, positive, or zero charges, respectively). Both lead chloride and lead bromide exhibit anionic conduction via halide ion vacancies up to high temperatures. In the intrinsic conductivity region the concentration of the halide ion vacancies is governed by Eq. (1), whereas Eq. (2) and (3) determine the concentration of 
the vacancies in the extrinsic region of the conductivity.

Previous work on the dielectric properties has been restricted mainly to measurements of the dielectric constant (13). No information was available about dielectric relaxation phenomena in lead halides. On account of the experience with the alkali halides and similar materials one would expect to be present, at least in principle, one or more of the following three types of dipole centres (14).

(i) Dipole centres as a result of the presence of diatomic or triatomic molecules or of offcentre ions.

(ii) Breckenridge type of centres, as a result of the formation of associates, e.g., between vacancies and impurities.

(iii) Electronic centres which have trapped electrons (or holes) in such a manner that the latter can still move between several equivalent positions. Their presence has been observed in some transition metal oxides and in smoky quartz. However, the existence of such a centre in halide compounds, to our knowledge, has been reported only once. It concerns the work of Grissom and Hartwig (15), who attributed the losses observed below $4.2^{\circ} \mathrm{K}$ in a $\mathrm{NaCl}$ crystal to a hopping motion of an electron between two neighbouring iron ions.

In general the reorientation of a dipole will be influenced strongly by the symmetry properties of its environment. The alkali, silver, and alkaline earth halides have as a common feature a highly symmetric crystal structure. This is in striking contrast to the structure of the lead halides. For that reason we are interested in the occurrence and behaviour of dipole centres in lead chloride and lead bromide.

\section{Dipole Centres, Observed in $\mathbf{P b C l}_{2}$ and $\mathbf{P b B r}_{2}$}

In order to investigate the presence of dipole centres of type (i) (see above) $\mathrm{PbCl}_{2}$ and $\mathrm{PbBr}_{2}$ crystals doped with $\mathrm{OH}^{-}$and $\mathrm{PbBr}_{2}$ crystals doped with $\mathrm{Be}^{2+}$ were grown (16). Within the frequency and temperature range at our disposal we did not detect losses which could be attributed to the presence of these dopants.
Nevertheless, these crystals did exhibit relaxation losses which appear to be of the same kind as we found in lead halide crystals both nominally pure and doped with several monovalent metals. We believe associates (as mentioned above under (ii)) to be responsible for the occurrence of these losses. The experimental procedure, the identification of the dipoles and the determination of the activation enthalpy have been described in a previous paper, to which we will refer as Paper A (17). These experiments are closely related to ionic conductivity experiments. Moreover, results from conductivity measurements are often very helpful for the interpretation of the losses. The results of both investigations have therefore been presented in Paper A together.

The results of the dielectric investigations may be summarized as follows.

(a) Associates like $\left(\mathrm{Me}_{\mathrm{Pb}} \cdot \mathrm{V}_{\mathrm{X}}\right)^{\times}$are formed which can be oriented with a relaxation time $\tau=\tau_{0} \exp H_{r} / k T$. We found $\tau_{0}=1.5 \times 10^{-13} \mathrm{~s}$ and $7.0 \times 10^{-11} \mathrm{~s}$ and $H_{r}=0.37 \mathrm{eV}$ and 0.20 $\mathrm{eV}$ for $\mathrm{PbCl}_{2}$ and $\mathrm{PbBr}_{2}$ crystals, respectively. The halide vacancy is supposed to be the mobile part of the dipole.

(b) In $\mathrm{PbBr}_{2}$ crystals doped with $\mathrm{CuBr}$ we found a relaxation process which we attributed to the motion of an electron between two neighbouring multivalent copper ions. The values of $\tau_{0}$ and $H_{r}$ are $3 \times 10^{-6} \mathrm{~s}$ and $28 \mathrm{meV}$, respectively. We may remark that this $\tau_{0}$ value is more like the values usually found in the transition metal oxides and in quartz than the value reported by Grissom and Hartwig (namely $4 \times 10^{-11} \mathrm{~s}$ ).

The remaining part of this paper will be devoted to a further analysis of the loss experiments presented in Paper A.

\section{Analysis of the Dielectric Susceptibility}

In general the concentration or the moment of the dipoles involved in a relaxation process can be derived from the susceptibility $\chi$ as given by the maximum value of the loss according to:

$$
\tan \delta_{\max }=2 \pi \chi / \varepsilon_{s}
$$

The value of $\varepsilon_{s}$ at temperature $T$ can be taken from Smakula's data (13) which have been 
confirmed by our experiments. Usually in such calculations one employs the relation:

$$
\chi=\beta N p^{2} / k T
$$

where $N$ denotes the number of dipoles per unit volume and $p$ the component of the dipole moment in the direction of the applied field. Let us for the time being omit the correction factor $\beta$ which accounts for local field corrections. We assume that owing to the rather rapid cooling of the sample during the experiment we are dealing with a frozen-in state. This may be argued from the fact that even close to the melting temperature (which implies large mobility) the vacancies can be frozen-in quite easily (18) and also from photodecompositon experiments (4) which indicate that below $200^{\circ} \mathrm{K}$ no mass transport takes place because of the very low mobility of the halide ion vacancies (19). Consequently we suppose $N$ to be constant.

Equation (4) implies that the product $\chi T$ should be independent of the temperature when $N$ does not vary with $T$. In Fig. 1 we plotted this product versus $T^{-1}$ for a lead chloride and a lead bromide crystal. We will confine ourselves to the case of dipoles related with associates. Contrary to the expectation $\chi T$ decreases very strongly with decreasing $T$. Considering $N$ a constant we must conclude that relation (4) does not pertain to our case.

It appears that this decreasing can be clarified by using the concept of localized

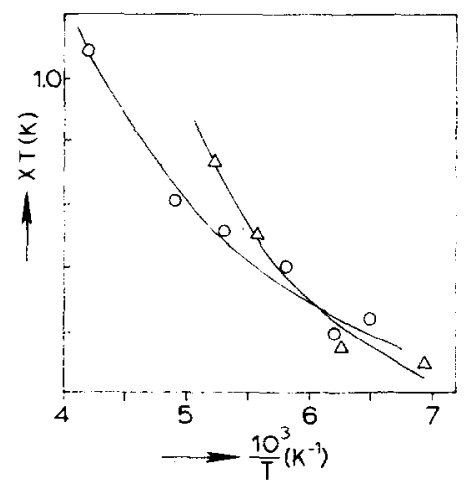

Fig. 1. The product $\chi T$ versus $T^{-1}$, plotted for a $\mathrm{PbCl}_{2}: \mathrm{BeCl}_{2}$ crystal $(O)$ and a $\mathrm{PbBr}_{2}: \operatorname{TiBr}$ crystal $(\Delta)$.

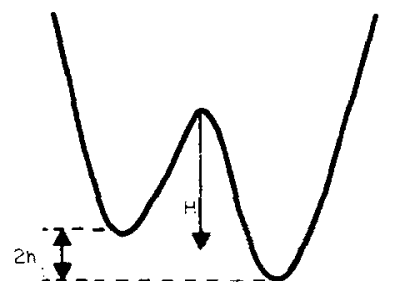

FIG. 2. One-dimensional asymmetric double well potential.

dipoles. We first want to discuss some of their properties.

\subsection{Some Properties of Localized Dipoles}

The use of expression (4) can be justified when the Fröhlich model for dipole relaxation, consisting of a symmetric double well potential in which a charge carrier moves (20), can be applied. This model can be generalized quite easily to the case of an asymmetric double well potential (Fig. 2). In such a model lowering of the temperature will be accompanied by an increasing localization of the charge carrier, i.e., considering the dynamical properties of the system - of the dipole, in the lowest well.

Calculation of the susceptibility (21) and the relaxation time in the case of localized dipoles yields:

$$
\chi=N p^{2} / k T \operatorname{sech}^{2} h / k T
$$

and

$$
\tau=\tau_{0} e^{\mathbf{H} / \mathbf{k T}} \operatorname{sech} h / k T
$$

where $h$ denotes the deviation from the average barrier height $H$.

In general one expects the existence of a distribution $y(h)$ of $h$ values, symmetric around the average value $\langle h\rangle=o$, with $\int y(h(d h=1$. In that case Eq. (5) should be replaced by

$$
\langle\chi\rangle=G \int_{-\infty}^{\infty} y(h) \operatorname{sech}^{2} h / k T d h
$$

and Eq. (6) in a similar way.

If the asymmetry is related to the structure of the ideal crystal one could expect only a few discrete $h$ values to be important. The most 
simple assumption for a distribution is then:

$$
y\left(h^{\prime}\right)=\frac{1}{2}\left[\delta\left(h^{\prime}-h\right)+\delta\left(h^{\prime}+h\right)\right] .
$$

The corresponding expressions for $\langle\chi\rangle$ and $\langle\tau\rangle$ are again the equations (5) and (6), respectively. The activation enthalpy is determined from the slope of the $\log \langle\tau\rangle$ versus $T^{-1}$ plot:

$$
\frac{d \log \langle\tau\rangle}{d T^{-1}}=\frac{H}{k}-\frac{h}{k} \tanh \frac{h}{k T} .
$$

In two limiting cases the plot will be a straight line: either if the asymmetry can be neglected or if it is large (i.e., $h \ll k T$ or $h>k T$ ). In the latter case the experimentally determined slope $H_{r}$ is given by:

$$
H_{r}=H-|h| \text {. }
$$

If the asymmetry arises, for instance, from internal strains one would expect $y(h)$ to be (at least roughly) of a Gaussian shape. The integrals, however, cannot be calculated by analytical methods for that case. The influence of such a distribution can nevertheless be described in an approximate way by introducing the following distribution:

$$
y\left(h^{\prime}\right)=\frac{1}{2 h} \quad \text { if } \quad\left|h^{\prime}\right| \leqslant h
$$

and zero elsewhere. (In fact, this is strictly analogous to the well known Fröhlich distribution for relaxation times (20).) The corresponding expression for $\langle\chi\rangle$ becomes:

$$
\langle\chi\rangle=\frac{N p^{2}}{k T} \frac{\tanh h / k T}{h / k T} .
$$

Only in the limiting case $h \ll k T$ the $\log \langle\tau\rangle$ versus $T^{-1}$ plot will be a straight line; in the opposite case $(h \gg k T)$ the slope is given by

$$
\frac{d \log \langle\tau\rangle}{d T^{-1}}=\frac{H}{k}-T \text {. }
$$

\subsection{Experimental Results}

It appears to be impossible to describe the experimental results as shown in Fig. 1 by a susceptibility which behaves according to Eq. (12). A satisfactory agreement, however, can be obtained between the experiments and a $\chi$ as given by Eq. (5). The drawn curves in Fig. 1 represent the functions $f\left(T^{-1}\right)=$ $A \operatorname{sech}^{2} h / k T$. The values of $h$, required for a good fit, are $33 \mathrm{meV}$ and $50 \mathrm{meV}$ for the doped $\mathrm{PbCl}_{2}$ and $\mathrm{PbBr}_{2}$ crystal, respectively. The value of $50 \mathrm{meV}$ has also been found in a second $\mathrm{PbBr}_{2}$ sample.

From these facts we may conclude that the mobile part of the dipole, i.e., the halide vacancy, moves in an asymmetric double well potential, the magnitude of the asymmetry being characteristic for the material. As a consequence the relaxation time ought to behave according to Eq. (6). On account of the fact that obviously $h>k T$, the plot of $\log \langle\tau\rangle$ versus $T^{-1}$ should be a straight line. This was indeed found as can be seen from Fig. 4 in Paper A.

From Eq. (10) the average barrier height $H$ can now be calculated. The results have been collected in Table $I$; in addition we have included the experimentally found values of the migration enthalpy $H_{m}$.

In order to calculate the number of dipoles $N$ with the help of Eq. (5) we have to know the magnitude of the dipole moment $p$ which can be estimated in some cases from crystal structure parameters. However, this turns out to be a rather complicated problem. As an example we have shown in Fig. 3 a (010) plane of $\mathrm{PbCl}_{2}$. One lead ion has been replaced by a monovalent metal ion, e.g., a $\mathrm{K}^{+}$ion; the projections of the nine surrounding chlorine positions on the plane have been denoted. The dipole, being a $\left(\mathrm{K}_{\mathrm{Pb}}^{\prime} \cdot \mathrm{V}_{\mathrm{C} 1}^{*}\right)^{\times}$associate, may be aligned in nine different directions. In principle

TABLE I

Some Dielectric RelaXation Parameters of $\mathrm{PbCl}_{2}$ AND $\mathrm{PbBr}_{2}$

\begin{tabular}{lcccc}
\hline & $H_{r}(\mathrm{eV})$ & $h(\mathrm{meV})$ & $H(\mathrm{cV})$ & $H_{m}(\mathrm{eV})$ \\
\hline $\mathrm{PbBr}_{2}$ & $0.20 \pm 0.02$ & $50 \pm 5$ & $0.25 \pm 0.02$ & $0.25 \pm 0.02$ \\
$\mathrm{PbCl}_{2}$ & $0.37 \pm 0.02$ & $33 \pm 5$ & $0.40 \pm 0.02$ & $0.35 \pm 0.02$ \\
\hline
\end{tabular}




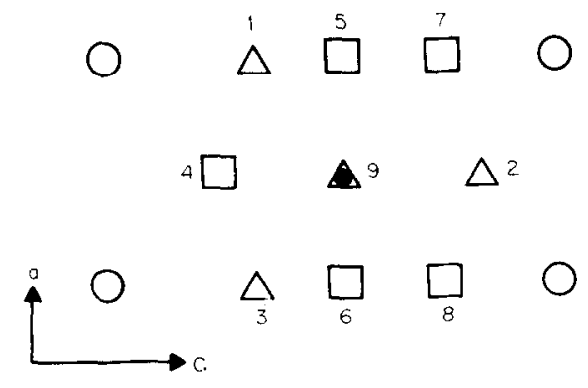

FIG. 3. The (010) plane through the lead ions in $\mathrm{PbCl}_{2}$ doped with $\mathrm{KCl}$. The electric field is applied along the $c$-axis. The positions labelled by $1,2,3$ or $4,5,6$ or $7,8,9$ denote the projections of chlorine positions approximately in, or slightly below or slightly above the plane, respectively. $0=\mathrm{Pb}^{2+} ; \bullet=\mathrm{K}^{+}$at a $\mathrm{Pb}^{2+}$ site; $\Delta$ and $\square$ indicate the projections of a $\mathrm{Cl}(1)$ and $\mathrm{Cl}(2)$ site, respectively.

there are 36 jumps of the vacancy between two chlorine positions leading to a reorientation. The situation outlined in Fig. 3 is characteristic for half of the lead ion sites. The remainder gives rise to the opposite case, i.e., the state which one gets by reflecting Fig. 3 with respect to the $a$-axis. In the case of $\left(\mathrm{A}_{\mathrm{Cl}}^{\prime} \cdot \mathrm{V}_{\mathrm{Cl}}^{*}\right)^{\times}$dipoles where $\mathrm{A}$ denotes a divalent anion, e.g., an oxygen ion, if any are present, the situation is analogous.

Inspection of the crystal structure quite generally yields:

1. Some jumps are not possible because of steric hindrance.

2. The reorientation can always occur by means of jumps between a $\mathrm{Cl}(1)$ and a $\mathrm{Cl}(2)$ site, i.e., between nonequivalent sites (leading possibly to localized dipoles).

3 . The reorientation may also occur by jumping between two $\mathrm{Cl}(1)$ or two $\mathrm{Cl}(2)$ sites, i.e., between equivalent sites (leading to "delocalized" dipoles).

Assuming that the jumps between all equivalent $\mathrm{Cl}(1)$ or $\mathrm{Cl}(2)$ sites or between nonequivalent sites occur with the characteristic frequencies $v_{e}(1), v_{e}(2)$ or $v_{n}$, respectively, we may expect three relaxation processes to be present, observable in general by the appearance of three loss peaks and distinguishable (at least partly) by an essentially different behaviour of the susceptibility $\chi(T)$.

\subsection{Discussion}

The above inspection of the crystal structure justifies our analysis of the susceptibility in terms of localized dipoles. First: the vacancy may indeed move between two nonequivalent sites; secondly: the presence of two kinds of $\mathrm{Pb}^{2+}$ sites agrees with the use of a distribution $y\left(h^{\prime}\right)$ as defined in Eq. (8). As pointed out above, losses due to delocalized dipoles (jumping between equivalent sites) should be present too. If jumping between equivalent sites would be easier than between nonequivalent sites $\left(v_{e}>v_{n}\right)$, such a loss should in principle become detectable by lowering the temperature. However, we did not observe it. In the opposite case $v_{e}<v_{n}$ the loss should become detectable by raising the temperature. The observation, however, is interfered by both an increasing contribution of the conductivity to the loss and a decrease of the susceptibility (because for delocalized dipoles $\chi$ varies as $T^{-1}$ ).

An analysis of the jump possibilities between equivalent sites indicates that the loss arising from jumping between $\mathrm{Cl}(2)$ sites will be much larger than the loss from jumping between $\mathrm{Cl}(1)$ sites. We may assume therefore in first instance that, when losses due to delocalized dipoles would be observed, they should be attributed to jumping between $\mathrm{Cl}(2)$ sites. This fact combined with the absence of such losses indicates that $v_{e}(2)<v_{n}$ and suggests that the deepest potential well in Fig. 2 is related to a $\mathrm{Cl}(2)$ (or $\mathrm{Br}(2)$ ) position.

For the magnitude of the dipole moment $p$ involved in the observed relaxation process we will take as a value 6 Debije (estimated from a crystal structure analysis) for both $\mathrm{PbCl}_{2}$ and $\mathrm{PbBr}_{2}$. Although this is a rather crude approximation, the use of a more accurate value is scarcely justified at the moment: the exact positions of the ions in the environment of the defect are unknown. We expect them to deviate from the ideal positions, because of the presence of an aliovalent ion at a $\mathrm{Pb}^{2+}$ (or $\mathrm{X}^{-}$) site. Furthermore, the lattice around the vacancy will relax (cf. Section 5 of Paper A). In general these effects may also account for possible small differences between migration and reorientation enthalpies.

If we now calculate the dipole concentration 
$N$ from Eq. (5) for the $\mathrm{PbCl}_{2}: \mathrm{BeCl}_{2}$ crystal we find as a result $N=3 \times 10^{19} \mathrm{~cm}^{-3}$.

From ionic conductivity experiments it is known (17) that the remainder impurity content is in the order of $10^{17} \mathrm{~cm}^{-3}$, as in undoped $\mathrm{PbCl}_{2}$. The explanation for the strong discrepancy between both values is in our opinion the omission of the local field correction factor $\beta$ in Eq. (5). Putting $\beta=1$ in the case of the alkali halides, is justified (22) by the fact that the vacancy associated with the impurity jumps from one lattice position to another which is crystallographically equivalent. However, this argument does not hold for the lead halides where the vacancy jumps between nonequivalent sites. The application of a local field correction therefore seems to be reasonable. However, a theoretical value of $\beta$ in our case is hard to obtain. If a Lorentz field correction would be applicable $\beta$ becomes about 220 , taking the average dielectric constant. Reducing the calculated value of $N$ by such a factor results in a value much more compatible with the impurity content. A similar situation arises in the case of the $\mathrm{PbBr}_{2}$ : $\mathrm{TIBr}$ crystal. The calculated value of $N$ is $4 \times 10^{20} \mathrm{~cm}^{-3}$ whereas $\left[\mathrm{Tl}_{\mathrm{Pb}}\right]=3$ to $5 \times 10^{18}$ $\mathrm{cm}^{-3}$. This implies that also in this case a local field correction seems to be necessary. Moreover this correction seems to be of the same order of magnitude as in the case of $\mathrm{PbCl}_{2}$.

We may remark that our analysis of the susceptibility confirms the assumption of a temperature independent dipole concentration. The same applies to the electronic dipole process in $\mathrm{PbBr}: \mathrm{CuBr}$ where the product $\chi T$ does not vary significantly with temperature. Assuming for the last mentioned process $p=6$ Debije and a local field correction $\beta=$ 200 the dipole concentration may be estimated to be about $10^{16} \mathrm{~cm}^{-3}$. This is consistent with the fact that the influence of divalent copper could be ignored in the ionic conductivity experiments.

Summarizing, we may conclude that the occurrence of localized dipoles, as observed in the behaviour of the susceptibility, and probably also the existence of a local field correction, can be understood from an inspection of the crystal structure, which clearly indicates the presence of nonequivalent sites. As a matter of fact it reflects the low site symmetry of the lead in the lead halides as compared to the site symmetries in many other halide compounds.

\section{Acknowledgments}

The authors wish to thank Dr. J. Schoonman for some helpful discussions and valuable criticism during the preparation of the manuscript. The investigations were performed as part of the research program of the "Stichting voor Fundamenteel Onderzoek der Materie" (F.O.M.) with financial support from the "Nederlandse Organisatie voor Zuiver-Wetenschappelijk Onderzoek" (Z.W.O.).

\section{References}

1. W. C. DE GruYter and J. Kerssen, J. Solid State Chem. 5 , 3 (1972).

2. B. Willemsen, Phys. Status Solidi (a) $11 \mathrm{~K}, 157$ (1972).

3. B. Willemsen, Phys. Status Solidi (a) 14, 565 (1972); K. J. DE VRIES, Thesis, University of Utrecht, 1965.

4. J. F. Verwey, J. Phys. Chem. Solids 31, 163 (1970).

5. Q. H. F. VReHEN AND J. Volger, Physica 31, 845 (1965); B. Willemsen, Phys. Status Solidi (a) 10, 183 (1972); J. KeRsSEN, W. C. DE GRUYTER, AND J. Volger, Physica (1973), in press.

6. K. J. De VRIES and J. H. VAN SANTEN, Physica 29, 482 (1963); 30, 2051 (1964).

7. J. Schoonman, J. Solid State Chem. 4, 466 (1972).

8. J. F. Verwey AND J. SchoOnMan, Physica 35, 386 (1967); B. Willemsen, J. Solid State Chem. 4, 567 (1971).

9. H. BRAEKKEN, Z. Krist. 83, 222 (1932).

10. W. Nieuwenkamp and J. M. Bijvoet, $Z$. Krist 84, 49 (1932).

11. R. W. G. Wrckoff: "Crystal Structures," Interscience Publishers, New York (1948).

12. F. A. KRöGER, "The Chemistry of Imperfect Crystals," North-Holland Publ. Co., Amsterdam (1964).

13. A. Smakula, M.I.T. Technical Report No. 6 (1965).

14. e.g., J. Volger, "Progress in Semiconductors," Vol. 4, p. 205, Heywood and Co. (1960); V. Narayanamurti and R. O. Poht, Rev. Mod. Phys. 42, 201 (1970).

15. D. Grissom and W. H. Hartwig, J. Appl. Phys. 37, 4784 (1966).

16. P. R. Boelens, Internal Report V-3181, Physisch Laboratorium Utrecht (1969). 
17. W. E. van den Brom, J. Schoonman, and J. H. W. DE WrT, J. Solid State Chem. 4, 475 (1972).

18. J. Schoonman, private communication.

19. W. C. De Gruyter and J. Schoonman, Phot. Sci. Eng. 17 (July 1973).

20. H. FRÖHLICH, "Theory of Dielectrics," University Press, Oxford (1958).
21. Equation (5) has also been used in some investigations into the dielectric properties of organic compounds: see R. J. Meakins in "Progress in Dielectrics," Vol. 3, p. 151, Heywood and Co., London (1961).

22. See for instance: A, B. Lidiard in "Molecular Mechanisms of Rate Processes in Solids" (A discussion of the Faraday Society 1957, p. 83). 